\title{
Triglycerides-to-HDL ratio as a new marker of endothelial dysfunction in obese prepubertal children
}

\author{
Tommaso de Giorgis ${ }^{1,2}$, M Loredana Marcovecchio ${ }^{1,2}$, Ilaria Di Giovanni', \\ Cosimo Giannini ${ }^{1,2}$, Valentina Chiavaroli ${ }^{1,2}$, Francesco Chiarelli ${ }^{1,2}$ \\ and Angelika Mohn ${ }^{1,2}$
}

${ }^{1}$ Department of Pediatrics, University of Chieti, Ospedale Policlinico, Via dei Vestini 5, 66100 Chieti, Italy and ${ }^{2}$ Clinical

Research Center, University of Chieti, Chieti, Italy
Correspondence should be addressed to A Mohn

Email

amohn@unich.it

\begin{abstract}
Objective: To investigate whether there is an association of the triglyceride-to-HDL cholesterol (TG:HDL-C) ratio with cardiovascular risk factors and early signs of vascular damage in obese prepubertal children. Design and methods: In 50 obese (27 boys, $7.8 \pm 1.4$ years) and 37 normal-weight (20 boys; $7.3 \pm 1.5$ years) prepubertal children, anthropometric measurements, oxidative stress markers (urinary isoprostanes (PGF2 $\alpha$ (prostaglandin F2 $\alpha$ )), soluble receptor for advanced glycation end-products (sRAGE)) and insulin sensitivity (homeostasis model assessment of insulin resistance (HOMA-IR) and whole-body insulin sensitivity index (WBISI)) were evaluated. Lipids profile was assessed and the TG:HDL-C ratio was calculated. In addition, high-resolution ultrasound was performed to assess carotid intima-media thickness (cIMT).

Results: Obese children showed significantly higher values of the TG:HDL-C ratio $(1.9 \pm 1.1$ vs $1.2 \pm 0.6, P=0.002)$ compared with controls. After dividing the population in tertiles of the TG:HDL-C ratio $(<1.04,1.04-1.67,>1.67)$, cIMT $(P=0.0003)$, and HOMA-IR $(P=0.0001)$ progressively increased from the lower to the upper tertile, whereas WBISI $(P=0.0003)$ and sRAGE $(P=0.05)$ progressively decreased. In a regression model, the TG:HDL ratio was significantly and positively associated with $\operatorname{CIMT}(r=0.493 ; P=0.0005)$. A cutoff point for TG:HDL-C ratio of 1.12 had $81 \%$ sensitivity and $49 \%$ specificity in the identification of children with cIMT values in the upper quartile (Area under the curve values from receiver operating characteristic curves $=0.633 \pm 0.065, P=0.045$ ).

Conclusion: This study confirms the reliability of the TG:HDL-C ratio as a useful marker of cardiovascular risk. Interestingly, our results underline that the TG:HDL-C ratio is directly related with early signs of vascular damage already present in
\end{abstract} prepubertal children.

\section{Introduction}

Childhood obesity is an important health problem that has reached epidemic proportions worldwide (1). Several lines of evidence have highlighted an alarming association between childhood obesity and the development of cardiovascular disease (2). Excess body weight during childhood leads to metabolic and inflammatory alterations, which in turn may induce changes in the arterial wall and contribute to the occurrence of cardiovascular events during adulthood $(2,3)$.

During the last years, several studies have shown that obese children and adolescents already present early signs of atherosclerosis, such as increased carotid intima-media thickness (cIMT) $(4,5)$. Many metabolic and inflammatory factors seem to be implicated in the pathogenesis of
(ㄷ) 2014 European Society of Endocrinology Printed in Great Britain
Published by Bioscientifica Ltd. 
atherosclerosis in obese children. In particular, insulin resistance (IR) represents an important link between obesity and the associated cardiovascular risk $(6,7)$, and it has been suggested as one of the first mechanisms involved in the development of endothelial dysfunction in obese youth $(5,8)$. In addition, oxidative stress and proinflammatory molecules, related to an increased adipose tissue, are additional players in the development of the atherosclerotic plaque (5).

Recently, there has been growing interest in the role of the triglyceride-to-HDL cholesterol (TG:HDL-C) ratio as a new emerging marker, which could predict subjects at increased risk of developing metabolic and cardiovascular complications $(9,10)$. In obese adults, the TG:HDL-C ratio is a useful marker for the early identification of subjects with IR and at risk of cardiovascular complications (11). With regards to the pediatric population, recently Giannini et al. (10) have found that the TG:HDL-C ratio was strongly associated with IR in obese children, and suggested that this marker may be used, along with other risk factors, to identify young subjects at increased cardiometabolic risk. In addition, Di Bonito et al. (12) showed that, in obese children and adolescents, the TG:HDL-C ratio is related to signs of cardiac remodeling, such as concentric left ventricular hypertrophy. However, up to now, no data are available on the role of the TG:HDL-C ratio as a potential marker of early vascular damage in obese children.

The aim of this study was to investigate whether there is an association between the TG:HDL-C ratio and cardiovascular markers as well as early signs of vascular damage in obese prepubertal children.

\section{Subjects and methods}

\section{Study population}

We recruited 50 obese prepubertal children (27 boys and 23 girls) who had been referred to the Obesity Clinic of the Department of Pediatrics, University of Chieti, Italy. All subjects were obese (BMI $>$ 95th percentile for the age and sex), but otherwise healthy. None had other chronic diseases (diabetes, endocrine disorders, hereditary diseases, or systemic inflammation) or were taking any medication. As a control group, we recruited 37 (20 boys and 17 girls) normal-weight prepubertal children comparable for age, gender, and pubertal stage, who were admitted in the Department of Paediatrics of the University of Chieti for minor diseases (trauma, orthopedic disease, etc.). Blood and urinary samples and anthropometric and instrumental measurements were taken only after complete recovery of those diseases.

The Ethics Committee of University of Chieti approved the study. Parental informed consent and child assent were obtained.

\section{Study methods}

A detailed medical and family history was obtained from all study participants and a complete physical examination was performed, including anthropometric measurements (height, weight, waist circumference (WC), and hip circumference (HC)), staging of puberty, and blood pressure measurements.

A fasting blood sample for measurement of lipid profile, insulin, glucose, and soluble receptor for advanced glycation end-products (sRAGE) was collected from all subjects, before starting an oral glucose tolerance test.

All subjects were asked to keep an overnight urine collection on the night preceding the study visit. On a second day, after an interval of 1-2 days, the study participants underwent an ultrasound assessment of the right and left cIMT.

\section{Anthropometric measurements}

Body weight was determined to the nearest $0.1 \mathrm{~kg}$ and height was measured with Harpenden stadiometer to the nearest $0.1 \mathrm{~cm}$. BMI was calculated as weight/height ${ }^{2}$ and expressed as $\mathrm{kg} / \mathrm{m}^{2}$. WC was measured at its smallest point between iliac crest and rib cage (13); HC was evaluated at its largest width over the greater trochanters. Height, weight, and BMI SDS were calculated based on the age and sex reference values for Italian children and using the LMS method (14).

In all subjects, pubertal stage was defined on the basis of breast development in girls and genital development in boys (15).

\section{Oral glucose tolerance test}

Subjects were seated for the test between 0800 and $0900 \mathrm{~h}$, after fasting overnight for at least $12 \mathrm{~h}$. After a plasma baseline sample for measurements of plasma glucose, insulin, and lipids, flavored glucose in a dose of $1.75 \mathrm{~g} / \mathrm{kg}$ body weight (up to maximum of $75 \mathrm{~g}$ ) was given orally, and blood samples were obtained every $30 \mathrm{~min}$ up to $120 \mathrm{~min}$ for the measurement of plasma glucose and insulin. 


\section{Indexes of IR}

We used homeostasis model assessment of IR (HOMA-IR) for the determination of IR: HOMA-IR was calculated with the formula: (fasting insulin $(\mathrm{mU} / \mathrm{l}) \times$ fasting glucose $(\mathrm{mmol} / \mathrm{l})) / 22.5$. In addition, we calculated whole-body insulin sensitivity index (WBISI) in order to estimate the insulin sensitivity: $(10000 /($ fasting glucose $\times$ fasting insulin $\times$ mean glucose concentration $\times$ mean insulin concentration) ${ }^{1 / 2}$ ).

\section{Biochemical analysis}

Plasma glucose level was determined using the glucose oxidase method, and plasma insulin was measured with two-site immunoenzymometric assay (AIA-PACK IRI, Tosoh, Tokyo, Japan). The limit of detection was $0.5 \mu \mathrm{U} / \mathrm{ml}$ with intra- and interassay coefficients of variation $(\mathrm{CV})<7 \%$ for quality control.

\section{Lipid analysis}

Serum total cholesterol (TC), HDL-C, and TG concentrations were determined by calorimetric enzymatic method. LDL cholesterol (LDL-C) was calculated according to the Friedewald formula (LDL-C $=\mathrm{TC}-\mathrm{HDL}-\mathrm{C}-$ $\mathrm{TG} / 5$ ). In addition, the TG:HDL-C ratio was calculated.

\section{Urinary isoprostanes}

Urine samples were added with the antioxidant 4-hydroxy-tempo (Sigma Chemical Co.) and multiple aliquot samples were stored at $-80^{\circ} \mathrm{C}$ until analysis. Urinary isoprostanes (PGF2 $\alpha$ (prostaglandin F2 $\alpha$ )) were evaluated in triplicate by a immunoenzymatic method (ELISA, Oxford Biomedical Research (Oxford, MI, USA), Enzyme Immunoassay for Urinary Isoprostane) (16).

\section{Soluble receptor for advanced glycation end-products}

Serum concentration of sRAGE was measured in duplicate by using the B-Bridge sRAGE ELISA Kit (which determines the total pool of all sRAGE; manufactured by Daiichi Fine Chemicals, Takaoka, Japan, and distributed by B-Bridge Int., San Francisco, CA, USA). The intra-assay CV for repeated sRAGE measurements ranged from 3.5 to $6.7 \%$ and from 3.2 to $7.1 \%$ respectively.

\section{Instrumental procedures}

Blood pressure $\bullet$ Blood pressure was measured in children by an investigator, using a validation protocol.
Systolic blood pressure (SBP) and diastolic blood pressure (DBP) were measured twice at the right arm after 10-min rest in supine position using a calibrated sphygmomanometer and average. The cuff size, which was based on the length and circumference of the upper arm, was chosen to be as large as possible without having the elbow skin crease obstructing the stethoscope. An inflatable bladder width that was at least $40 \%$ of the arm circumference at a point midway between the olecranon and the acromion and that was such a length as to cover $80-100 \%$ of the circumference of the arm was used. Hypertension was defined as blood pressure values above the 95th percentile for height, age, and sex (17).

Carotid ultrasonography $\bullet$ High-resolution B-mode ultrasonography of the right and left carotid arteries was performed with a linear $14 \mathrm{mHz}$ transducer from Philips Sonos. Children were examined in the supine position with the head turned slightly to the left and right. The common, internal, and external carotid arteries were identified by combined B-mode and Color-Doppler ultrasound examinations. A careful search was performed to obtain an optimal visualization of the vessel wall demonstrating the typical double lines representing the intima-media layer. cIMT was defined as the distance between the leading edge interface of the far wall and the leading edge of the median adventitia interface of the far wall, as described previously $(5,18)$.

The ultrasonic protocol requires the visualization of the near and far wall of the right and left common carotid, internal carotid arteries, and bifurcation in three different projections: anterior, lateral, and posterior, for a total of $\sim 15$ carotid segments per patient. All procedures were performed according to recent recommendations proposed by the American Heart Association (18).

Three determinations of cIMT were conducted and these three determinations were averaged (mean cIMT). During the ultrasonography valuations, images were frozen and printed. The measurements were performed by the same examiner who was blinded to the participants' case status and risk factors level.

\section{Statistical analysis}

Analyses were performed using SPSS version 16 Software for Windows (SPSS, Inc.). Data were analyzed for normality using the Kolmogorov-Smirnov test, and logtransformed to normal distributions wherever necessary to allow use of parametric tests. All data are expressed as 
mean \pm s.D. or median (interquartile range) unless otherwise specified. Two-tailed significance was set to $P<0.05$.

Unpaired $t$-test and $\chi^{2}$ test were used to assess differences between the two study groups for continuous and categorical variables respectively.

After categorizing subjects according to tertiles of the TG:HDL-C ratio, differences in cIMT and other parameters across these tertiles were evaluated by one-way ANOVA test. Tukey's test was used for post-hoc comparison of means between each pair of groups. Adjustment for potential confounding factors (BMI, age, sex, blood pressure, and LDL-C) was performed using analysis of covariance.

Receiver operating characteristic (ROC) curve analysis was performed to estimate a threshold of TG:HDL-C ratio that was able to identify the subjects in the upper quartile of cIMT. The optimal cutoff point for TG:HDL-C ratio was obtained using the Youden index (maximum (sensitivity + specificity -1$)$ ). A logistic regression analysis was performed to assess the odds ratio (OR) of subjects with high TG:HDL-C ratio for showing cIMT in the upper quartile, and age and sex were used as covariates. Results were expressed as OR with 95\% CI.

Pearson's correlation was performed to evaluate the relationship between TG:HDL-C ratio and each variable relating to cardio-metabolic risk (BMI, blood pressure, WC, lipid profile, HOMA-IR, WBISI, PGF2 $\alpha$, and sRAGE).

Multiple linear regression analysis was performed to assess the possible independent association between cIMT and TG:HDL-C ratio, after adjusting for other factors, using two different models: model A, where cIMT was the dependent variable and BMI SDS, sRAGE, HOMA-IR, PGF2 $\alpha$, age, and sex were the independent variables and model $\mathrm{B}$, where cIMT was the dependent variable and BMI SDS, WBISI, sRAGE, PGF2 $\alpha$, age, and sex were the independent variables.

\section{Results}

\section{Anthropometric characteristics}

The general characteristics and levels of biochemical parameters of the obese and normal-weight prepubertal children are reported in Table 1 . The two groups were similar for age, sex, and pubertal stage. As expected, weight, weight SDS, BMI, and BMI SDS were significantly higher in obese children than in controls (all $P<0.05$ ). No differences were found in SBP and DBP between the two groups (SBP, $P=0.306$; DBP, $P=0.379$ ).
Table 1 Baseline clinical characteristics and levels of biochemical parameters. Data are mean \pm s.D.

\begin{tabular}{|c|c|c|c|}
\hline & $\begin{array}{l}\text { Obese pre- } \\
\text { pubertal } \\
\text { children } \\
(n=50)\end{array}$ & $\begin{array}{c}\text { Prepubertal } \\
\text { controls } \\
(n=37)\end{array}$ & $\boldsymbol{P}$ \\
\hline \multicolumn{4}{|c|}{ Anthropometric measurements } \\
\hline Age (years) & $7.8 \pm 1.4$ & $7.3 \pm 1.5$ & 0.08 \\
\hline Gender & $27 \mathrm{M} / 23 \mathrm{~F}$ & $20 \mathrm{M} / 17 \mathrm{~F}$ & 0.320 \\
\hline Height (cm) & $131.7 \pm 12.4$ & $128.3 \pm 15.4$ & 0.302 \\
\hline Height SDS & $1.2 \pm 0.9$ & $1.2 \pm 1.0$ & 0.853 \\
\hline Weight (kg) & $42.5 \pm 1.3$ & $28.6 \pm 7.3$ & 0.0004 \\
\hline BMI $\left(\mathrm{kg} / \mathrm{m}^{2}\right)$ & $24.2 \pm 4.3$ & $16.9 \pm 1.6$ & 0.0006 \\
\hline BMI SDS & $2.63 \pm 0.59$ & $-0.06 \pm 0.73$ & 0.0002 \\
\hline WC $(\mathrm{cm})$ & $74.1 \pm 12.9$ & $55.6 \pm 6.5$ & 0.0005 \\
\hline $\mathrm{HC}(\mathrm{cm})$ & $80.7 \pm 10.03$ & $64.9 \pm 9.6$ & 0.0005 \\
\hline $\mathrm{SBP}(\mathrm{mmHg})$ & $104 \pm 8$ & $102 \pm 9$ & 0.306 \\
\hline $\mathrm{DBP}(\mathrm{mmHg})$ & $66 \pm 8$ & $66 \pm 8$ & 0.379 \\
\hline \multicolumn{4}{|l|}{ Insulin resistance } \\
\hline Fasting insulin $(\mu \mathrm{U} / \mathrm{ml})$ & $10.4 \pm 6.4$ & $3.9 \pm 1.5$ & 0.0001 \\
\hline Fasting glycemia (mg/dl) & $89 \pm 8$ & $85 \pm 7$ & 0.32 \\
\hline HOMA-IR & $2.2 \pm 1.3$ & $0.8 \pm 0.3$ & 0.0002 \\
\hline WBISI & $7.9 \pm 4.6$ & $15.4 \pm 6.6$ & 0.0006 \\
\hline \multicolumn{4}{|l|}{ Lipid profile } \\
\hline Total cholesterol (mg/dl) & $164 \pm 12$ & $165 \pm 14$ & 0.892 \\
\hline HDL cholesterol (mg/dl) & $52 \pm 12$ & $62 \pm 13$ & 0.06 \\
\hline LDL cholesterol (mg/dl) & $95 \pm 16$ & $88 \pm 13$ & 0.114 \\
\hline Triglyceride (mg/dl) & $91 \pm 38$ & $75 \pm 30$ & 0.059 \\
\hline TG:HDL-C ratio & $1.9 \pm 1.1$ & $1.2 \pm 0.6$ & 0.002 \\
\hline \multicolumn{4}{|l|}{ Oxidant status } \\
\hline sRAGE $(p g / m l)$ & $946.9 \pm 425.3$ & $1481.6 \pm 589.4$ & 0.0001 \\
\hline PGF2 $\alpha(\mathrm{ng} / \mathrm{ml})$ & $7.8 \pm 3.2$ & $1.7 \pm 0.8$ & 0.0001 \\
\hline \multicolumn{4}{|l|}{ cIMT } \\
\hline Right cIMT (mm) & $0.42 \pm 0.06$ & $0.29 \pm 0.10$ & 0.0004 \\
\hline Left cIMT (mm) & $0.43 \pm 0.06$ & $0.32 \pm 0.07$ & 0.0003 \\
\hline Mean cIMT (mm) & $0.42 \pm 0.06$ & $0.31 \pm 0.07$ & 0.0003 \\
\hline
\end{tabular}

$M$, male; F, female; WC, waist circumference; HC, hip circumference; WHR, waist-to-hip circumference ratio; SBP, systolic blood pressure; DBP, diastolic blood pressure; HOMA-IR, homeostasis model assessment of insulin resistance; WBISI, whole-body insulin sensitivity index; TG:HDL-C, triglyceridesto-HDL cholesterol ratio; PGF2 $\alpha$, urinary isoprostanes; sRAGE, soluble receptor for advanced glycation end-products; cIMT, intima-media thickness.

\section{Indices of IR/insulin sensitivity}

No differences were found between the two groups in fasting glycemia, whereas obese children had higher levels of fasting insulin than normal-weight children $(P=0.0001)$. In addition, obese children presented a higher HOMA-IR $(P=0.0002)$ and a lower WBISI $(P=0.0006)$ compared with control children (Table 1$)$.

\section{Oxidant-antioxidant status}

Levels of PGF2 $\alpha$ were higher in obese children than in controls $(P=0.0001)$, whereas sRAGE levels were lower in obese than in normal-weight children $(P=0.0001)$ (Table 1). 


\section{Lipid profile}

No significant differences were found between two groups in TC, LDL-C and HDL-C, and TG (TC, $P=0.89$; HDL-C, $P=0.06$; LDL-C, $P=0.114$; and TG, $P=0.059$ ), whereas the TG:HDL-C ratio was significantly higher in obese than in normal-weight children $(1.9 \pm 1.1$ vs $1.2 \pm$ $0.6, P=0.002$ ) (Table 1 ).

\section{Intima-media thickness}

Obese children presented a significantly higher cIMT, both on right and left sides, compared with normal-weight children ( $P=0.0004, P=0.0003$ respectively), as well as higher mean values of the left and the right carotid arteries (cIMT mean $P=0.0003$ ) (Table 1 ).

\section{Association between TG:HDL-C ratio and cardiovascular risk factors}

To evaluate the influence of the TG:HDL-C ratio on IR, oxidative stress, and cIMT, subjects were categorized according to tertiles of the TG:HDL-C ratio (1st tertile, $<1.04$; 2nd tertile, 1.04-1.67; and 3rd tertile, >1.67). No significant differences were found in age and sex across the tertiles of the TG:HDL-C ratio. BMI SDS and WC progressively increased from the lower to the upper tertile of the TG:HDL-C ratio $(P=0.0001$ and $P=0.0007$ respectively). HOMA-IR $(P=0.0001)$ progressively increased from the lower to the upper tertile, whereas WBSI $(P=0.0003)$ and sRAGE $(P=0.05)$ progressively decreased (Table 2$)$.

Table 2 cIMT, metabolic parameters, and levels of oxidative status across tertiles of the TG:HDL-C ratio. Data are mean \pm s.D.

\begin{tabular}{|c|c|c|c|c|}
\hline & \multicolumn{3}{|c|}{ Tertiles of TG:HDL-C ratio } & \multirow[b]{2}{*}{$\boldsymbol{P}$} \\
\hline & $\begin{array}{c}\text { 1st tertile } \\
(<1.04)\end{array}$ & $\begin{array}{c}\text { 2nd tertile } \\
(1.04-1.67)\end{array}$ & $\begin{array}{l}\text { 3rd tertile } \\
\quad(>1.67)\end{array}$ & \\
\hline $\operatorname{Sex}(M / F)$ & $13 \mathrm{M} / 15 \mathrm{~F}$ & $15 \mathrm{M} / 13 \mathrm{~F}$ & $18 \mathrm{M} / 13 \mathrm{~F}$ & 0.231 \\
\hline Age (years) & $7.6 \pm 1.3$ & $7.2 \pm 0.9$ & $7.9 \pm 0.8$ & 0.342 \\
\hline BMI SDS & $1.14 \pm 0.49$ & $1.86 \pm 1.30$ & $2.39 \pm 1.27$ & $0.0001 *,+$ \\
\hline WC $(\mathrm{cm})$ & $65.5 \pm 1.2$ & $60 \pm 9.0$ & $78 \pm 1.4$ & $0.0007 *,+$ \\
\hline HOMA-IR & $1.4 \pm 1.2$ & $1.0 \pm 0.4$ & $2.5 \pm 1.3$ & $0.0001 *,+$ \\
\hline WBISI & $11.9 \pm 6$ & $12.9 \pm 5.9$ & $6.4 \pm 4.9$ & $0.0003^{*}+\dagger$ \\
\hline sRAGE (pg/ml) & $1350 \pm 759$ & $1101 \pm 423$ & $1000 \pm 444$ & $0.05^{*}$ \\
\hline PGF2 $\alpha(\mathrm{ng} / \mathrm{ml})$ & $5.9 \pm 4.3$ & $6.4 \pm 3.8$ & $6.2 \pm 3.6$ & 0.891 \\
\hline cIMT (mm) & $0.35 \pm 0.1$ & $0.37 \pm 0.1$ & $0.43 \pm 0.1$ & $0.0003 *,+$ \\
\hline
\end{tabular}

Significant values by post-hoc analysis: *3rd tertile vs 1 st tertile and ${ }^{\dagger} 3$ rd tertile vs 2nd tertile. WC, waist circumference; HOMA-IR, homeostasis model assessment of insulin resistance; WBISI, whole-body insulin sensitivity index; TG:HDL-C, triglycerides-to-HDL cholesterol ratio; PGF2 $\alpha$, urinary isoprostanes; SRAGE, soluble receptor for advanced glycation endproducts; cIMT, intima-media thickness.

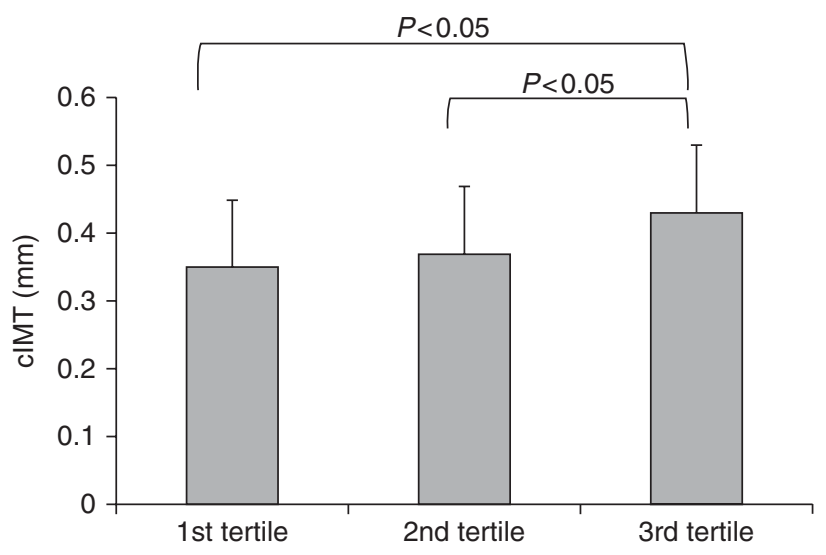

Figure 1

Changes in cIMT according to tertiles of the TG:HDL-C ratio.

Interestingly, also cIMT progressively increased moving across tertiles ( $P=0.0003$; Table 2 and Fig. 1$)$.

Post-hoc analysis showed significant differences in the parameters between the upper tertile when compared with both the lower and the middle tertiles, but not between the lower and middle tertiles (Table 2). These changes in metabolic and cardiovascular markers persisted after dividing the study population in to obese and normalweight children (Tables 3 and 4).

\section{ROC curve}

A ROC curve was calculated to individuate a value of the TG:HDL-C ratio that was able to identify children with cIMT in the upper quartile. The AUC-ROC for the ability of TG:HDL-C ratio to predict values of cIMT in the highest quartile was significant $(0.633 \pm 0.065$, $P=0.045$ ). In particular, we found that the cutoff point of 1.12 for the TG:HDL-C ratio had a $81 \%$ sensitivity and $49 \%$ specificity in the identification of prepubertal children with values of cIMT in the upper quartile. Children with a TG:HDL-C ratio higher than 1.12 had an OR of 4.775 (95\% CI 1.503-15.174, $P=0.008)$, having their cIMT in the upper quartile after adjusting for age and sex.

\section{Relationship between TG:HDL-C and CIMT}

A positive and statistically significant correlation was found between the TG:HDL-C ratio and all cardiometabolic parameters: BMI SDS $(r=0.414, P=0.0005)$, HOMA-IR $(r=0.345, P=0.001)$, WBISI $(r=-0.346$, $P=0.001), \quad$ PGF $2 \alpha \quad(r=0.924, \quad P=0.01), \quad$ and sRAGE $(r=-0.414, P=0.347)$. In addition, a significant and 
Table 3 cIMT, metabolic parameters, and levels of oxidative status across tertiles of the TG:HDL-C ratio in normal weight children. Data are mean \pm s.D.

\begin{tabular}{|c|c|c|c|c|}
\hline & \multicolumn{3}{|c|}{ Tertiles of TG:HDL-C ratio } & \multirow[b]{2}{*}{$\boldsymbol{P}$} \\
\hline & $\begin{array}{c}\text { 1st tertile } \\
(<0.97)\end{array}$ & $\begin{array}{c}\text { 2nd tertile } \\
(0.97-1.38)\end{array}$ & $\begin{array}{c}\text { 3rd tertile } \\
(>1.38)\end{array}$ & \\
\hline HOMA-IR & $0.66 \pm 0.11$ & $0.90 \pm 0.27$ & $1.0 \pm 0.35$ & $0.011 *$ \\
\hline WBISI & $17.83 \pm 9.03$ & $14.38 \pm 4.22$ & $14.14 \pm 5.38$ & 0.308 \\
\hline PGF2 $\alpha$ & $1.23 \pm 0.21$ & $1.76 \pm 0.82$ & $1.97 \pm 0.81$ & $0.031 *$ \\
\hline sRAGE & $1526.0 \pm 583.7$ & $1469.2 \pm 975.3$ & $1481.6 \pm 589.6$ & 0.952 \\
\hline cIMT (mm) & $0.25 \pm 0.05$ & $0.33 \pm 0.04$ & $0.35 \pm 0.06$ & $0.002^{*}+\dagger$ \\
\hline
\end{tabular}

Significant values by post-hoc analysis: ${ }^{*} 3$ rd tertile vs 1 st tertile and ${ }^{\dagger} 3$ rd tertile vs 2 nd tertile. HOMA-IR, homeostasis model assessment of insulin resistance; WBISI, whole-body insulin sensitivity index; TG:HDL-C, triglycerides-to-HDL cholesterol ratio; PGF2 $\alpha$, urinary isoprostanes; SRAGE, soluble receptor for advanced glycation end-products; cIMT, intima-media thickness.

positive correlation was also found between the TG: HDL-C ratio and cIMT ( $r=0.493, P=0.0005$; Fig. 2$)$.

In order to investigate the potential independent contribution of the TG:HDL-C ratio on cIMT, a multiple stepwise regression analysis was performed. In the model A, where we considered as independent variables BMI SDS, HOMA-IR, TG:HDL-C ratio, sRAGE, PGF2 $\alpha$, age, and sex, cIMT was independently and positively associated only with TG:HDL-C ratio and PGF2 $\alpha$ (Table 5). In the second model (model B), where the independent variables were BMI SDS, WBISI, TG:HDL-C ratio, sRAGE, PGF2 $\alpha$, age, and sex, cIMT was significantly associated with TG:HDL-C ratio, PGF2 $\alpha$, and WBISI, whereas age, sex, BMI SDS, and sRAGE were not related (Table 5).

\section{Discussion}

In this study, we have found that obese prepubertal children had an increased TG:HDL-C ratio compared

Table 4 cIMT, metabolic parameters, and levels of oxidative status across tertiles of the TG:HDL-C ratio in obese children. Data are mean \pm s.D.

\begin{tabular}{|c|c|c|c|c|}
\hline & \multicolumn{3}{|c|}{ Tertiles of TG:HDL-C ratio } & \multirow[b]{2}{*}{$\boldsymbol{P}$} \\
\hline & $\begin{array}{c}\text { 1st tertile } \\
(<1.14)\end{array}$ & $\begin{array}{l}\text { 2nd tertile } \\
(1.14-2.25)\end{array}$ & $\begin{array}{l}\text { 3rd tertile } \\
\quad(>2.25)\end{array}$ & \\
\hline HOMA-IR & $1.56 \pm 0.97$ & $2.05 \pm 1.55$ & $2.67 \pm 1.21$ & $0.02 *$ \\
\hline WBISI & $10.2 \pm 4.61$ & $8.49 \pm 2.62$ & $5.73 \pm 2.41$ & $0.005 *$ \\
\hline PGF2 $\alpha$ & $5.79 \pm 2.15$ & $7.48 \pm 1.93$ & $8.48 \pm 3.68$ & $0.01 *,+$ \\
\hline sRAGE & $1526.0 \pm 5.83$ & $1460.2 \pm 975.3$ & $1481.6 \pm 589.6$ & 0.179 \\
\hline cIMT (mm) & $0.39 \pm 0.05$ & $0.43 \pm 0.03$ & $0.44 \pm 0.06$ & 0.014 * \\
\hline
\end{tabular}

Significant values by post-hoc analysis: * 3 rd tertile vs 1 st tertile and ${ }^{\dagger} 3$ rd tertile vs 2 nd tertile. HOMA-IR, homeostasis model assessment of insulin resistance; WBISI, whole-body insulin sensitivity index; TG:HDL-C, triglycerides-to-HDL cholesterol ratio; PGF2 $\alpha$, urinary isoprostanes; sRAGE, soluble receptor for advanced glycation end-products; cIMT, intima-media thickness. with normal-weight peers, and that this ratio was associated with well-known cardiovascular risk factors. Even more interesting was the finding of a significant association between the TG:HDL-C ratio and early abnormalities in the arterial wall, such as increased cIMT.

During the last years, several studies have clearly demonstrated the role of atherogenic dyslipidemia in the pathogenesis of atherosclerosis in obese subjects $(10,12$, $19,20)$. The hallmark of atherogenic dyslipidemia is represented by decreased levels of HDL-C associated with increased TG and normal or minimally elevated levels of LDL-C (20). However, in adults with an increased cardiometabolic risk, the individual values of TG, HDL-C, and LDL-C do not always reflect their overall cardiovascular risk, whereas the combination of TG and HDL-C in a single ratio seems to have a better predictive power for cardiovascular disease (21). In line with these data, in this study, we found that obese prepubertal children presented no differences in terms of TG and HDL-C levels compared with normal-weight peers, whereas the TG:HDL-C ratio was significantly increased in obese children.

Recent guidelines for the clinical approach to obese patients recommend that the TG:HDL-C ratio should be used to define the impaired metabolic status and chronic inflammation in these subjects (22). Recently, some studies have reported that even in the pediatric population, the TG:HDL-C ratio is related to IR and chronic inflammation $(10,12,23)$. Accordingly with these findings, our results confirm the already reported association between TG:HDL-C ratio, IR status, and chronic inflammation, and highlight the value of the TG:HDL-C

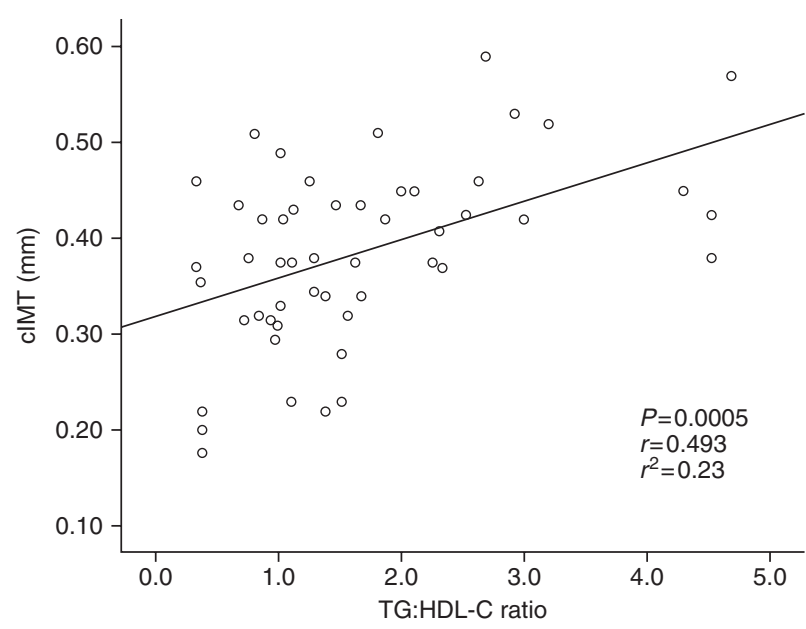

Figure 2

Association between cIMT and TG:HDL-C ratio. 
Table 5 Relationship between CIMT, TG:HDL-C, and other main parameters.

\begin{tabular}{|c|c|c|c|}
\hline & $\beta$ & $\boldsymbol{P}$ & $\begin{array}{c}R^{2} \\
\text { adjusted }\end{array}$ \\
\hline \multicolumn{4}{|c|}{$\begin{array}{l}\text { Dependent variable } \\
\text { CIMT }\end{array}$} \\
\hline \multicolumn{4}{|c|}{ Independent variables } \\
\hline PGF2 $\alpha$ & $\begin{array}{c}A-11, \text { age, } \\
0.576\end{array}$ & 0.0002 & 0.236 \\
\hline TG:HDL-C & 0.490 & 0.0005 & 0.303 \\
\hline \multicolumn{4}{|c|}{ Model B (BMI SDS, sRAGE, age, and gender) } \\
\hline PGF2 $\alpha$ & 0.576 & 0.0002 & 0.323 \\
\hline TG:HDL-C & 0.490 & 0.0005 & 0.561 \\
\hline WBISI & -0.230 & 0.003 & 0.601 \\
\hline
\end{tabular}

CIMT, intima-media thickness; TG:HDL-C, triglycerides-to-HDL cholesterol ratio; HOMA-IR, homeostasis model assessment of insulin resistance; WBISI, whole-body insulin sensitivity index; PGF2 $\alpha$, urinary isoprostanes; SRAGE, soluble receptor for advanced glycation end-products.

ratio as a marker of the cardio-metabolic and inflammatory status already during the prepubertal period. Overall, our data confirmed the role of IR and chronic inflammation in the pathogenesis of endothelial dysfunction. Our results are of interest mainly in the light of recent findings suggesting a role of the TG:HDL-C ratio in the identification of children and adults with higher concentration of small dense LDL and smaller LDL particle size, which are directly implicated in the pathogenesis of atherosclerosis (24). Furthermore, in a recent study Yang et al. (25) have reported that the severity of coronary artery stenosis was associated with the TG:HDL-C ratio. In a recent study, Di Bonito et al. (12) have proposed that the TG:HDL-C ratio is a useful index for identifying signs of early cardiac remodeling, such as left ventricular hypertrophy, even in obese children and adolescents. In this present study, we tried to extend the association between the TG:HDL-C ratio and cardiovascular disease, by assessing its relationship with cIMT, a vascular component significantly affected by excess body weight (5). During the last decade, cIMT has been proposed as one of the more feasible, direct, and non-invasive methods, detecting preclinical signs of arterial wall dysfunction in the pediatric population $(26,27)$. In this study, we showed that the TG:HDL-C ratio is an additional independent factor associated with cIMT, therefore providing a further line of evidence for a role of the TG:HDL-C ratio in the cardiovascular risk that was able to discriminate subjects with more marked signs of early atherosclerosis as indicated by higher values of cIMT. It needs to be acknowledged that in our study population, values of the TG:HDL-C ratio were not particularly high when compared with previous studies performed in obese youth $(10,23)$. However, our study population was made only of prepubertal and Caucasian children, and this could explain differences in TG:HDL-C compared with other studies, where also adolescents and a mixture of ethnic groups were studied. These could reflect the well-known influences of puberty and ethnicity on insulin sensitivity and cardio-metabolic parameters.

Some limitations of this study need to be acknowledged. In particular, the cross-sectional study design does not allow proving causality between the TG:HDL-C ratio and increased cIMT. In addition, the small sample size and the clinical-based design limit the generability of the results. Therefore, further larger and prospective studies are required to confirm our preliminary findings. Another study limitation might be the use of cIMT as a marker of early vascular damage instead of flow-mediated dilatation and arterial distensibility. However, several studies have validated cIMT and extensively applied it.

In conclusion, our findings support the role of the TG:HDL-C ratio as a useful marker, related to cardiovascular risk factors and early signs of vascular damage, and reiterate the concept that early signs of cardiovascular disease are already detectable in obese prepubertal children.

\section{Declaration of interest}

The authors declare that there is no conflict of interest that could be perceived as prejudicing the impartiality of the research reported.

\section{Funding}

This research did not receive any specific grant from any funding agency in the public, commercial or not-for-profit sector.

\section{Author contribution statement}

$\mathrm{T}$ de Giorgis researched and analyzed the data and wrote the manuscript; M L Marcovecchio wrote, reviewed, and edited the manuscript; I Di Giovanni analyzed the data and wrote the manuscript; C Giannini and $\checkmark$ Chiavaroli researched the data and edited the manuscript; $F$ Chiarelli reviewed and edited the manuscript; and A Mohn had the idea for the study, reviewed, edited the manuscript, and contributed to the discussion. All authors are the guarantors of this work and, as such, had full access to all the data in the study and took responsibility for the integrity of the data and the accuracy of the data analysis.

\section{Acknowledgements}

The authors would like to thank the contribution of Dr A Scarinci of the Department of Cardiology of University of Chieti for the high-resolution B-mode ultrasonography of the right and left carotid arteries. 


\section{References}

1 Han JC, Lawlor DA \& Kimm SY. Childhood obesity. Lancet 2010375 1737-1748. (doi:10.1016/S0140-6736(10)60171-7)

2 Daniels SR. Obesity in the pediatric patient: cardiovascular complications. Progress in Pediatric Cardiology 200112 161-167. (doi:10.1016/ S1058-9813(00)00069-2)

3 Juonala M, Magnussen CG, Berenson GS, Venn A, Burns TL, Sabin MA, Srinivasan SR, Daniels SR, Davis PH, Chen W et al. Childhood adiposity, adult adiposity, and cardiovascular risk factors. New England Journal of Medicine 2011365 1876-1885. (doi:10.1056/NEJMoa1010112)

4 Leite A, Santos A, Monteiro M, Gomes L, Veloso M \& Costa M. Impact of overweight and obesity in carotid intima-media thickness of Portuguese adolescents. Acta Paediatrica 2012101 e115-e121. (doi:10.1111/j.1651-2227.2011.02503.x)

5 Giannini C, de Giorgis T, Scarinci A, Ciampani M, Marcovecchio ML, Chiarelli F \& Mohn A. Obese related effects of inflammatory markers and insulin resistance on increased carotid intima media thickness in pre-pubertal children. Atherosclerosis 2008197 448-456. (doi:10.1016/ j.atherosclerosis.2007.06.023)

6 Caprio S. Insulin resistance in childhood obesity. Journal of Pediatric Endocrinology \& Metabolism 200215 (Suppl 1) 487-492.

7 Weiss R \& Kaufman FR. Metabolic complications of childhood obesity: identifying and mitigating the risk. Diabetes Care 200831 (Suppl 2) S310-S316. (doi:10.2337/dc08-s273)

8 De Fronzo RA. The effect of insulin on renal sodium metabolism. A review with clinical implications. Diabetologia 198121 165-171.

9 Quijada Z, Paoli M, Zerpa Y, Camacho N, Cichetti R, Villarroel V, Arata-Bellabarba G \& Lanes R. The triglyceride/HDL-cholesterol ratio as a marker of cardiovascular risk in obese children; association with traditional and emergent risk factors. Pediatric Diabetes 20089 464-471. (doi:10.1111/j.1399-5448.2008.00406.x)

10 Giannini C, Santoro N, Caprio S, Kim G, Lartaud D, Shaw M, Pierpont B $\&$ Weiss R. The triglyceride-to-HDL cholesterol ratio: association with insulin resistance in obese youths of different ethnic backgrounds. Diabetes Care 201134 1869-1874. (doi:10.2337/dc10-2234)

11 Salazar MR, Carbajal HA, Espeche WG, Leiva Sisnieguez CE, Balbín E, Dulbecco CA, Aizpurúa M, Marillet AG \& Reaven GM. Relation among the plasma triglyceride/high-density lipoprotein cholesterol concentration ratio, insulin resistance, and associated cardio-metabolic risk factors in men and women. American Journal of Cardiology 2012109 1749-1753. (doi:10.1016/j.amjcard.2012.02.016)

12 Di Bonito P, Moio N, Scilla C, Cavuto L, Sibilio G, Sanguigno E, Forziato C, Saitta F, Iardino MR, Di Carluccio C et al. Usefulness of the high triglyceride-to-HDL cholesterol ratio to identify cardiometabolic risk factors and preclinical signs of organ damage in outpatient children. Diabetes Care 201235 158-162. (doi:10.2337/dc11-1456)

13 D'Adamo E, Giannini C, Chiavaroli V, de Giorgis T, Verrotti A, Chiarelli F \& Mohn A. What is the significance of soluble and endogenous secretory receptor for advanced glycation end products in liver steatosis in obese pre-pubertal children? Antioxidants \& Redox Signaling 201114 1167-1172. (doi:10.1089/ars.2010.3719)
14 Cacciari E, Milani S, Balsamo A, Spada E, Bona G, Cavallo L, Cerutti F, Gargantini L, Greggio N, Tonini G et al. Italian cross-sectional growth charts for height, weight and BMI (2 to $20 \mathrm{yr}$ ). Journal of Endocrinological Investigation 200629 581-593.

15 Biro FM. Puberty. Adolescent Medicine: State of the Art Reviews 200718 425-433.

16 Roberts II \& Morrow JD. Measurement of F2-isoprostanes as an index of oxidative stress in vivo. Free Radical Biology \& Medicine 200028 505-513. (doi:10.1016/S0891-5849(99)00264-6)

17 National High Blood Pressure Education Program Working Group on High Blood Pressure in Children and Adolescents. The fourth report on the diagnosis, evaluation, and treatment of high blood pressure in children and adolescents. Pediatrics 2004114 555-576. (doi:10.1542/ peds.114.2.S2.555)

18 Amato M, Montorsi P, Ravani A, Oldani E, Galli S, Ravagnani PM, Tremoli E \& Baldassarre D. Carotid intima-media thickness by B-mode ultrasound as surrogate of coronary atherosclerosis: correlation with quantitative coronary angiography and coronary intravascular ultrasound findings. European Heart Journal 200728 2094-2101. (doi:10.1093/eurheartj/ehm244)

19 Arca M, Pigna G \& Favoccia C. Mechanisms of diabetic dyslipidemia: relevance for atherogenesis. Current Vascular Pharmacology 201210 684-686. (doi:10.2174/157016112803520864)

20 Kavey RE \& Mietus-Snyder M. Beyond cholesterol: the atherogenic consequences of combined dyslipidemia. Journal of Pediatrics 2012161 977-979. (doi:10.1016/j.jpeds.2012.07.034)

21 Kim-Dorner SJ, Deuster PA, Zeno SA, Remaley AT \& Poth M. Should triglycerides and the triglycerides to high-density lipoprotein cholesterol ratio be used as surrogates for insulin resistance? Metabolism 2010 59 299-304. (doi:10.1016/j.metabol.2009.07.027)

22 Lau DC, Douketis JD, Morrison KM, Hramiak IM, Sharma AM \& Ur E. 2006 Canadian clinical practice guidelines on the management and prevention of obesity in adults and children. CMAJ: Canadian Medical Association Journal 2007176 S1-S13. (doi:10.1503/cmaj.061409)

23 Musso C, Graffigna M, Soutelo J, Honfi M, Ledesma L, Miksztowicz V, Pazos M, Migliano M, Schreier LE \& Berg GA. Cardiometabolic risk factors as apolipoprotein $\mathrm{B}$, triglyceride/HDL-cholesterol ratio and C-reactive protein, in adolescents with and without obesity: crosssectional study in middle class suburban children. Pediatric Diabetes 201112 229-234. (doi:10.1111/j.1399-5448.2010.00710.x)

24 Burns SF, Lee SJ \& Arslanian SA. Surrogate lipid markers for small dense low-density lipoprotein particles in overweight youth. Journal of Pediatrics 2012161 991-996. (doi:10.1016/j.jpeds.2012.06.013)

25 Yang D, Liu X \& Xiang M. The correlation between lipids ratio and degree of coronary artery stenosis. High Blood Pressure \& Cardiovascular Prevention 201118 53-56. (doi:10.2165/11593480-000000000-00000)

26 Reinehr T \& Wunsch R. Intima media thickness-related risk factors in childhood obesity. International Journal of Pediatric Obesity 20116 (Suppl 1) 46-52. (doi:10.3109/17477166.2011.590199)

27 Yang EY \& Nambi V. Ultrasound imaging of carotid intima-media thickness: an office-based tool to assist physicians in cardiovascular risk assessment. Current Atherosclerosis Reports 201113 431-436. (doi:10.1007/s11883-011-0200-5)

Received 29 May 2013

Revised version received 3 October 2013

Accepted 28 October 2013 